\title{
A structured assessment on the perceived attributes of sustainable agricultural practices: a study for the Malaysian vegetable production sector
}

\begin{abstract}
Individual farmers subjectively perceive the attributes of green agricultural innovations and their subsequent benefits. Qualifying this knowledge is important, but past efforts have largely been unsystematic. This systematic study assesses the structure of perceived attributes of sustainable agricultural practices. Through a survey of Malaysian vegetable farmers, the results of confirmatory factor analysis indicate that the most robust structure consists of four attributes. The four important attributes are relative advantage, compatibility, complexity and trialability. Among them, measures are required to link noneconomic benefits to profitability to the attribute of relative advantage. Our work represents a guide to assist change agents to evaluate farmer perceptions at different locations systematically. This structured method will help effectively to design and promote sustainable agriculture and other green agricultural innovations.
\end{abstract}

Keyword: Green agricultural innovations; Attributes; Perceptions; Sustainable agricultural practices; Malaysia 